\title{
Position control for haptic device based on discrete-time proportional integral derivative controller
}

\author{
Nguyen Van Tan ${ }^{1}$, Khoa Nguyen Dang ${ }^{2}$, Pham Duc Dai ${ }^{3}$, Long Vu Van \\ ${ }^{1}$ Faculty of Engineering-Technology, Thu Dau Mot University, Bình Dương, Vietnam \\ ${ }^{2}$ Faculty of Electrical and Electronic Engineering, Phenikaa University, Hanoi, Vietnam \\ ${ }^{3}$ Department of Control Engineering and Automation, Thuyloi University, Hanoi, Vietnam \\ ${ }^{4}$ KiKai Tech., Hanoi, Vietnam
}

\section{Article Info \\ Article history: \\ Received Jan 2, 2021 \\ Revised Jun 23, 2021 \\ Accepted Jul 2, 2021}

\section{Keywords:}

Haptic

Novint Falcon

PID controller

Position controller

\begin{abstract}
Haptic devices had known as advanced technology with the goal is creating the experiences of touch by applying forces and motions to the operator based on force feedback. Especially in unmanned aerial vehicle (UAV) applications, the position of the end-effector Falcon haptic sets the velocity command for the UAV. And the operator can feel the experience vibration of the vehicle as to the acceleration or collision with other objects through a forces feedback to the haptic device. In some emergency cases, the haptic can report to the user the dangerous situation of the UAV by changing the position of the end-effector which is be obtained by changing the angle of the motor using the inverse kinematic equation. But this solution may not accurate due to the disturbance of the system. Therefore, we proposed a position controller for the haptic based on a discrete-time proportional integral derivative (PID) controller. A Novint Falcon haptic is used to demonstrate our proposal. From hardware parameters, a Jacobian matrix is calculated, which combines with the force output from the PID controller to make the torque for the motors of the haptic. The experiment was shown that the PID has high accuracy and a small error position.
\end{abstract}

This is an open access article under the CC BY-SA license.

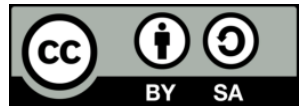

\section{Corresponding Author:}

Khoa Nguyen Dang

Faculty of Electrical and Electronic Engineering, Phenikaa University

To Huu Road, Yen Nghia, Hanoi 12116, Vietnam

Email: khoa.nguyendang@phenikaa-uni.edu.vn

\section{INTRODUCTION}

The automation field had a sharp increase in the amount of robotic industrialization, many autonomous mobile robots had been developed such as unmanned aerial vehicles (UAVs) and unmanned ground vehicles (UGVs). These robots are to support the human in many difficult missions and working in various environments, those tasks can be rescued scans, spaces, surgery or military. In general, almost the architecture of an autonomous robot is divided into two parts as the master part and the slave part. The slave side includes the human operator, computer, and controller devices which were directed interaction with humans. The master side includes the robot frame and the actuators integrated with a system of sensors such as the Lidar sensor, visual camera, and optical sensor. In this model, the human operator either sends the command to the master side via a wired or wireless network.

Normally, the human operator only sees the UAV and environment on the monitor based on the sensor and the visual feedback. Therefore, the tactile information needs to append to improve the feeling of the human operator. The haptic technology had seemed a key by the purpose is aim to bring more experience feeling about the robot working environment to the operator controller. For example, the personal haptic 
interface mechanism (PHANToM) [1], [2] and Novint Falcon [3], [4] devices enable are used to control the manipulator as well as feel the environment around the workspace of the robot. With similar functions of the two above devices, we selected the Novint Falcon to develop the control algorithms.

Novint Falcon haptic was used in many research and real applications. For example, the haptic presented the interaction forces between the excavator and soil [5], control position and velocity for the 3DOF manipulator [6], make position control for the 6DOF Denso VM6083 robot arm [7]. The realistic force feedback in cardiac surgery [8], [9]. Falcon haptic also integrated with a laser sensor to applied in a wheelchair with the purpose is support blind people [10], and the nuclear industry [11]. For some applications used the haptic to make feedback force to the user to help avoid obstacles is as in [12], [13]. In the UAV field, the end-effector of Falcon haptic is used to set velocity command for the UAV [14], [15], and the force feedback to haptic is to help the user avoiding obstacles that closed the UAV [16]-[18]. In some critical cases, the end-effector position of the Falcon is used for changing the angle of UAV motors using the inverse kinematic equation [19].

To apply the algorithms to the haptic device, the kinematic structure and dynamic configuration needs to be carefully considered as in some previous researches [20]-[23]. Herein, the inverse kinematic is expressed for calculating the angle of motors based on the know the end-effector position. In this case, the dynamic model is to determine the torques of the motors based on the Jacobian and force applied to the grip. It is easy to see that if we know the target position of the end-effector then we could control the angle of each motor correspond with this position. However, the system often exits more disturbance the motor disturbance (motor load, stiffness and damping), environmental conditions and error of mechanical. Therefore, the inverse kinematic will not provide an accurate position if only uses the equation by the angle motors. Therefore, the controller is designed to maintain the stability as well as the accuracy of the system. The proportional integral derivative (PID) controller [24], [25] is widely used to control the mechanism in industrial with the loop feedback, which calculates the error between the desired value and feedback value, and then it makes the control signal to the mechanism. Especially, PID control is useful to control the motion of the system [26]. In addition, the microchip and the personal computer need the sampling time to get the measured value from the system to make the feedback signal. Thus, this paper is aiming to develop a position controller which is to increment the accuracy of the position control for the end-effector position of the haptic device based on a discrete-time PID controller.

The paper is organized as follows: firstly, we are going to present the model configuration and the invest kinematic of the Novint Falcon haptic device in section 2. Secondly, the dynamics configuration as the Jacobian matrix is described in section 3 for obtained the actuator torques based on the end-effector trajectory. Then, the details of the proposed controller are presented in section 4 . After that, in section 5 we experimented with the position controller with an attitude and heading reference system (AHRS) sensor integrated with the grip to estimate the end-effector position, the result had demonstrated the performance of the proposed controller. Finally, the conclusions are given in section 6 .

\section{NOVINT FALCON HAPTIC DEVICE}

The geometry of the Novint Falcon was developed by Novint Technologies Company [27] as shown in Figure 1 [21]. The model design of Falcon has included three parts such as a moving platform, a base platform and three parallel linkages to connect the two above platforms. Three motors are mounted on the base platform. The operator can manually control the robot by moving the end-effector in the Falcon haptic and three internal motors could be made some force to the operator.

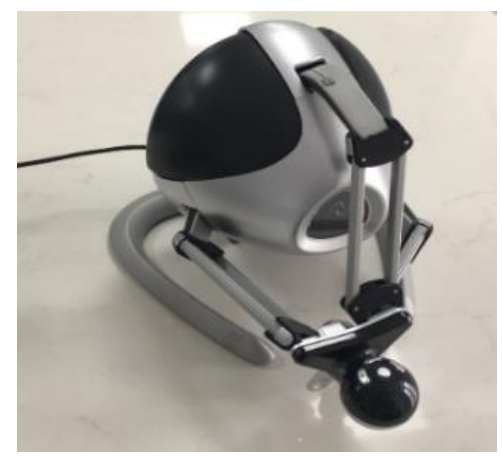

Figure 1. Novint falcon haptic hardware 
Figures 2 and 3 represent one leg and base of the Novint Falcon haptic, respectively. A reference coordinate $X Y Z$ is attached at point $O$ which is denoted the center of the base platform. The radius of the base platform is $r$ and the distance from the end-effector to the highest joint $\mathrm{E}$ is denoted $c$. The length of the associated between joints $A B, B C, C D$ and $D E$ are $a, e, b$ and $d$, respectively. The Novint Falcon dimensions are shown detail in Table 1 . The end-effector position denoted by point $P$ in the $X Y Z$ fixed coordinate frame and a local coordinate frame UVW are attached to the fixed point $A$. Three angles $\theta_{1 i}, \theta_{2 i}, \theta_{3 i} \in R^{3}$ with $i=(1,2,3)$ represent each joint $A, B$ and $C$, respectively.

Table 1. The dimension of parameters in Novint Falcon [28]

\begin{tabular}{cc}
\hline Parameters & Value $(\mathrm{mm})$ \\
\hline $\mathrm{a}$ & 60.0 \\
$\mathrm{~b}$ & 102.5 \\
$\mathrm{c}$ & 15.7 \\
$\mathrm{~d}$ & 11.5 \\
$\mathrm{e}$ & 11.5 \\
$\mathrm{f}$ & 26.2 \\
$\mathrm{~g}$ & 27.9 \\
$\mathrm{r}$ & 36.6 \\
$\mathrm{~s}$ & 27.2 \\
\hline
\end{tabular}

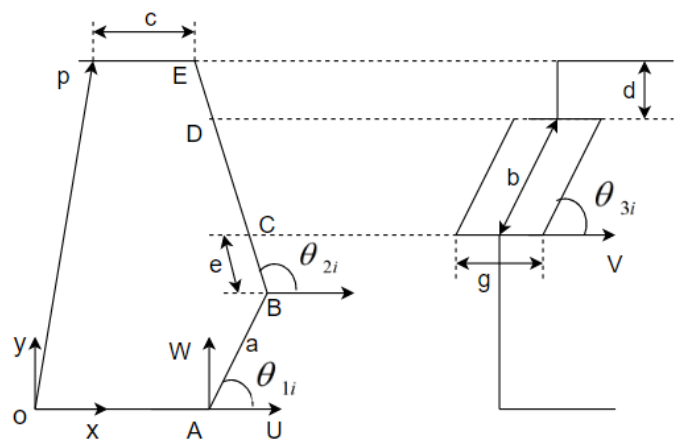

Figure 2. Structure of a linkage in Novint Falcon haptic

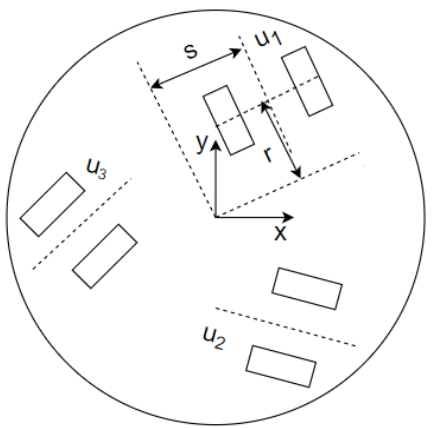

(a)

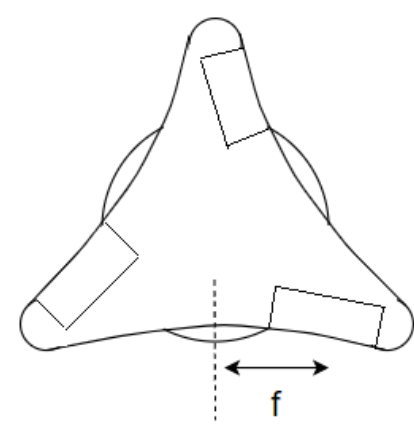

(b)

Figure 3. Novint Falcon platform (a) base platform, and (b) moving platform

The inverse kinematic is to calculate the three angles $\theta_{1 i}, \theta_{2 i}, \theta_{3 i}$ of the motors using the known location of the end-effector $P\left(P_{x}, P_{y}, P_{z}\right)$ which can present in the $U V W$ coordinate frame by the homogeneous transformation matrix as in (1).

$$
\left[\begin{array}{l}
P_{u i} \\
P_{v i} \\
P_{w i}
\end{array}\right]=\left[\begin{array}{ccc}
\cos \varphi_{i} & \sin \varphi_{i} & 0 \\
-\sin \varphi_{i} & \cos \varphi_{i} & 0 \\
0 & 0 & 1
\end{array}\right]\left[\begin{array}{l}
P_{x} \\
P_{y} \\
P_{z}
\end{array}\right]+\left[\begin{array}{l}
-r \\
0 \\
0
\end{array}\right]
$$


where the $\varphi_{i}$ is the angle from the center of Novint Falcon haptic to each axis $i=(1,2,3)$, which has the values as $\frac{7 \pi}{12}, \frac{-\pi}{12}$ and $\frac{-9 \pi}{12}$, respectively.

The angle actuators $\theta_{1 i}, \theta_{2 i}, \theta_{3 i}$ can be obtained by [15]:

$$
\begin{aligned}
& \theta_{1 i}=2 \operatorname{atan}\left(y_{1 i}\right) \\
& \theta_{2 i}=a \cos \left(\frac{P_{u i}-a \cos \theta_{1 i}+c}{d+e+b \sin \theta_{3 i}}\right) \\
& \theta_{3 i}= \pm\left(\frac{P_{v i}+f}{b}\right)
\end{aligned}
$$

with term $y_{1 i}$ can be determined from (5).

$$
l_{2 i} y_{1 i}^{2}+l_{1 i} y_{1 i}+l_{0 i}=0
$$

In which

$$
\begin{aligned}
& l_{0 i}=P_{w i}^{2}+P_{u i}^{2}+2 c p_{u i}-2 a p_{u i}-b^{2} \sin \left(\theta_{3 i}\right)^{2}-2 b e \sin \left(\theta_{3 i}\right)-2 b d \sin \left(\theta_{3 i}\right) \\
& -2 d e-2 a c+a^{2}+c^{2}-d^{2}-e^{2} \\
& l_{1 i}=-4 a p_{w i} \\
& l_{2 i}=P_{w i}^{2}+P_{u i}^{2}+2 c p_{u i}+2 a p_{u i}-b^{2} \sin \left(\theta_{3 i}\right)^{2}-2 b e \sin \left(\theta_{3 i}\right)-2 b d \sin \left(\theta_{3 i}\right) \\
& -2 d e+2 a c+a^{2}+c^{2}-d^{2}-e^{2}
\end{aligned}
$$

Following (2), (3) and (4), the position of the end-effector could be obtained by controlling the angle of the motors. However, the system often has disturbances that come from the noise of motors, and the environment. The inverse kinematic cannot provide accurately the position of the end-effector which could have trouble if it combines with the robotic systems. Therefore, in this paper, we propose the position control for the haptic device based on the discrete-time PID.

\section{DYNAMICS CONFIGURATION}

The Jacobian basic function can be defined as in (9).

$$
x=J q
$$

Where $\mathrm{x}$ is end-effector position and orientation; $q$ is the set of joint angles; $J$ is the Jacobian matrix. Let's define as in (10), (11) [17],

$$
\begin{aligned}
& J=\left[\begin{array}{lll}
J_{11} & J_{12} & J_{13} \\
J_{21} & J_{22} & J_{23} \\
J_{31} & J_{32} & J_{33}
\end{array}\right] ; \dot{q}=\left[\begin{array}{lll}
\dot{\theta}_{11} & \dot{\theta}_{12} & \dot{\theta}_{13}
\end{array}\right]^{T} ; \text { and } \dot{x}=\left[\begin{array}{lll}
v_{x} & v_{y} & v_{z}
\end{array}\right]^{T} \\
& J_{1 i}=-\frac{\cos \left(\phi_{i}\right) \cos \left(\theta_{2 i}\right) \sin \left(\theta_{3 i}\right)-\sin \left(\phi_{i}\right) \cos \left(\theta_{3 i}\right)}{\operatorname{asin} \theta_{3 i} \sin \left(\theta_{1 i}-\theta_{2 i}\right)} \\
& J_{2 i}=-\frac{\sin \left(\phi_{i}\right) \cos \left(\theta_{2 i}\right) \sin \left(\theta_{3 i}\right)+\cos \left(\phi_{i}\right) \cos \left(\theta_{3 i}\right)}{\operatorname{asin} \theta_{3 i} \sin \left(\theta_{1 i}-\theta_{2 i}\right)} \\
& J_{3 i}=-\frac{\sin \left(\theta_{2 i}\right) \sin \left(\theta_{2 i}\right)}{\operatorname{asin} \theta_{3 i} \sin \left(\theta_{1 i}-\theta_{2 i}\right)}
\end{aligned}
$$


with $i, j=(1,2,3)$ and $\theta_{j i}$ are in (2), (3) and (4). Applying formula of

$$
\tau=J^{T} F
$$

where $F$ is the vector combining end-effector forces and moments; $J$ is Jacobian matrix; $\tau$ is actuator torques. Therefore, the actuator torques can be obtained by the force of the end-effector.

\section{PROPOSED DISCRETE-TIME PID CONTROLLER}

In this section, a discrete-time PID controller is developed to drive the position of the Novint Falcon haptic device, which has the structure in Figure 4.

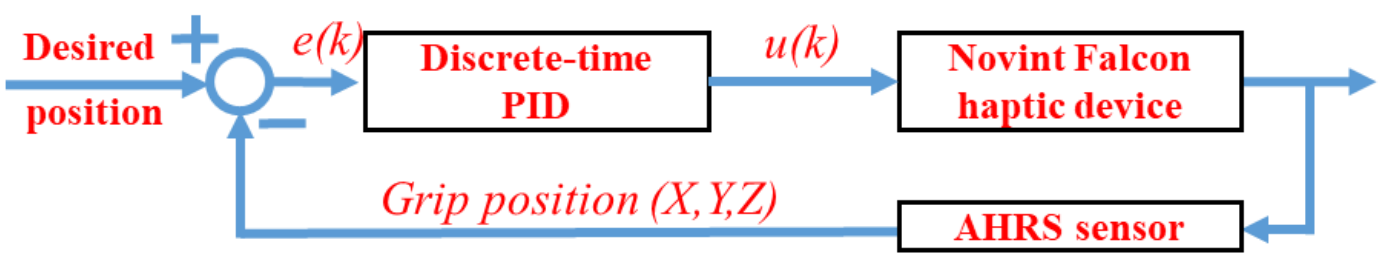

Figure 4. The diagram of position control proposal

Let's consider the continuous PID form in (12).

$$
u(t)=K_{p} e(t)+K_{i} \int_{0}^{t} e(\tau) d \tau+K_{d} \frac{d e(t)}{d t}
$$

Where the error $e(t)$ is defined by subtraction between the desired input value and the measured output value, $\mathrm{u}(\mathrm{t})$ is the controller output. Three parameters $K_{p}, K_{d}$, and $K_{i}$ are defined as proportional gain, derivative gain and integral gain, respectively.

Talking the $\mathrm{Z}$ transform of (12), one has

$$
U(z)=\left(K_{p}+K_{i} \frac{T_{S}}{2} \frac{z+1}{z-1}+K_{d} \frac{z-1}{z T_{S}}\right) E(z)=\frac{a+b z^{-1}+c z^{-2}}{1-z^{-1}}
$$

in which $\mathrm{Z}\left(\frac{d e(t)}{d t}\right)=\frac{z-1}{z} \frac{1}{T_{s}} E(z) ; \mathrm{Z}\left(\int_{0}^{t} e(\tau) d \tau\right)=\frac{z+1}{z-1} \frac{T_{s}}{2} E(z) ; a=K_{p}+K_{i} \frac{T_{s}}{2}+\frac{K_{d}}{T_{s}} \quad b=-K_{p}+K_{i} \frac{T_{s}}{2}-2 \frac{K_{d}}{T_{s}}$; $c=\frac{K_{d}}{T_{S}}$ and $T_{s}$ is the sampling period.

From (13), we have (14).

$$
U(z)=U(z) z^{-1}+a E(z)+b z^{-1} E(z)+c z^{-2} E(z)
$$

The time-domain of (14) can be presented as in (15). From (15), the PIDz can be applied to any system with the gains $K_{p}, K_{i}, K_{d}$ and sampling time $T_{s}$.

$$
u(k)=u(k-1)+a e(k)+b e(k-1)+c e(k-2)
$$

\section{EXPERIMENT AND RESULTS}

The experiment is performed to demonstrate the proposed position control, which includes the Novint Falcon haptic device connected to one desktop PC via the USB connection. The PC is installed in the Linux OS, the AHRS sensor is integrated with the grip to estimate the end-effector position to configure the input/output for the Novint device. Herein, the input is the force and the output is the position of the endeffector. The configuration of the experiment is shown in Figure 5. 
The discrete-time PID controller in Sec. 4 is a built-in Linux OS PC using the C++ program. In all simulations, we selected the sampling time $T_{s}=0.01[\mathrm{~s}]$. And the gains of a PID controller can be obtained by the trial and error method. By using this method, the $K_{d}$ and $K_{i}$ are set to zero for the first time. We increase the $K_{p}$ to the response becomes faster and the output of the loop oscillates. This step needs careful because the system could unstable if the $\mathrm{K}_{\mathrm{p}}$ is very large. After that, $\mathrm{K}_{i}$ is increased to stop the oscillations and reduce the steady-state error but the overshoot may appear. In the last step, $K_{d}$ is increased to cancel the error which is created by $\mathrm{K}_{\mathrm{i}}$. Finally, we can select the gain of the PID controller as $K_{p}=1.5, K_{d}=5, K_{i}=0.0013$. To prove the performance of the proposed controller, two scenarios are implemented in cases without the disturbance and with disturbance to the end-effector by the human forces.

Linux PC

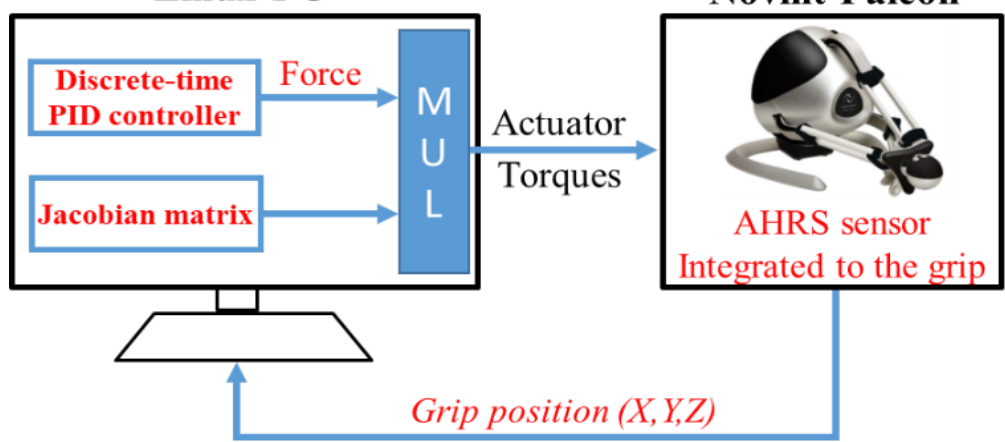

Figure 5. The configuration of experiment

In the first scenario, the initial of Novint Haptic is random position as $P_{x}=1.5[\mathrm{~cm}], P_{y}=-5.7[\mathrm{~cm}]$, $P_{z}=4.3[\mathrm{~cm}]$ and the desired position is $P_{x r}=2.5[\mathrm{~cm}], P_{y r}=-2.5[\mathrm{~cm}], P_{z r}=4.0[\mathrm{~cm}]$. As a result in Figure 6, the position of haptic could meet the desired position at $1.2[\mathrm{~s}]$ and keep stability during the time working which can show very detail in Figure 7 . Therein, all position errors $\left(e_{i}=P_{i r}-P_{i}\right.$ where $\left.i=x, y, z\right)$ come to zeros and maintain at this value. These results show that the discrete-time PID proposed control can provide a solution for controlling the end-effector position to any desired trajectory with high accuracy and ensure the stability system.

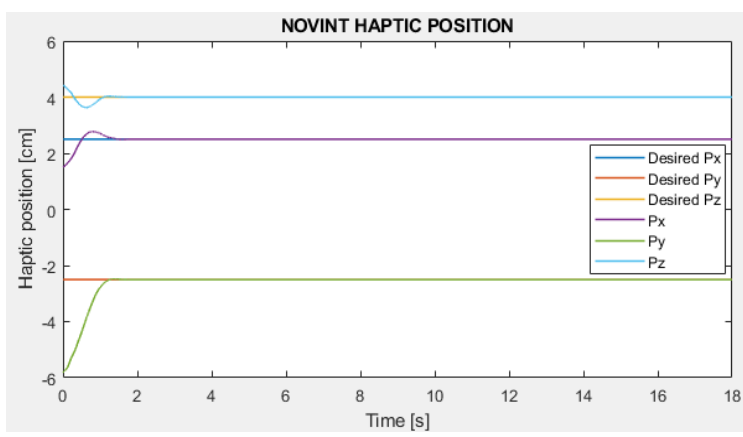

Figure 6. The position of end-effector without disturbance

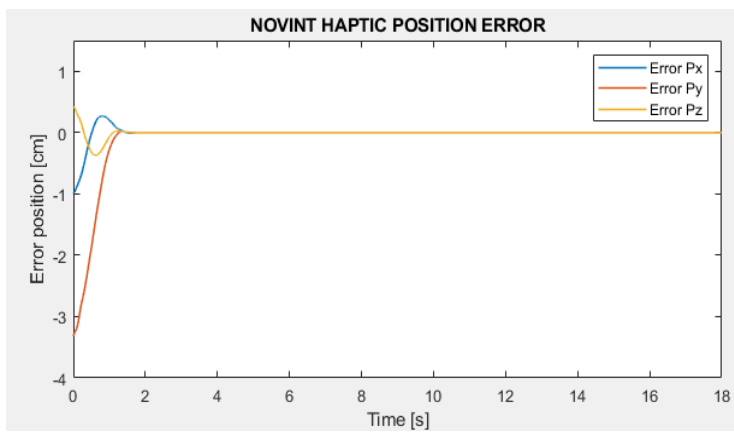

Figure 7. The error position of end-effector without disturbance

In the second scenario, a disturbance from human force is effected to the end-effector in the random time at $6.5[s], 11[s], 14.9[s], 19[s], 22[s]$ and $26.5[s]$ to all directions of the grip. The result in Figure 8 showed that the discrete-time PID can maintain the stability of the position end-effector haptic to the desired position notwithstanding the disturbance from any direction. The detailed result is shown in Figure 9 demonstrated that although the disturbances in this situation are quite large, the proposed controller still maintains the stability for the haptic device. The results proved convincingly that the position control performance could be ensured by using our proposed control with large noises and disturbances in environment. 


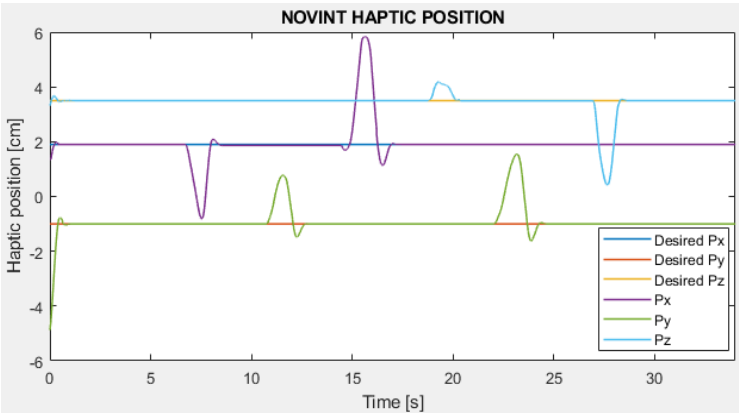

Figure 8. The position of end-effector with external forces

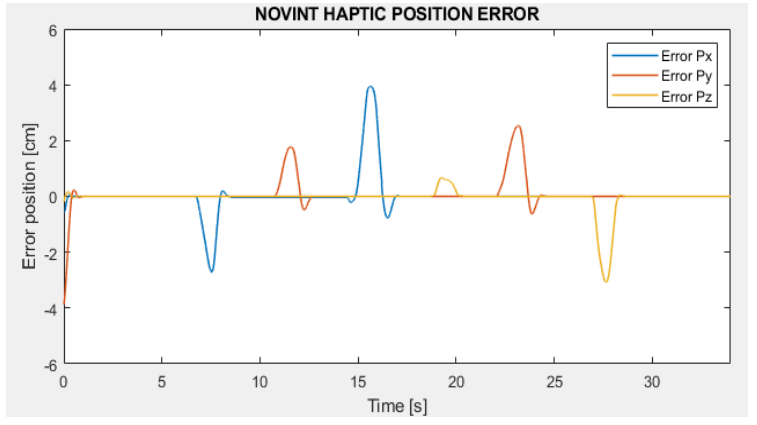

Figure 9. The error position of end-effector with external forces

\section{CONCLUSION}

This paper presents the discrete-time PID controller to drive the end-effector position of Novint Falcon haptic. Herein, the inverse kinematic and dynamic models are carefully considered and show some limitations of the control position. Instead of using the inverse kinematic equation to take the end-effector to go to the desired point, the proposed position control is suggested based on the PID controller. As a result, the grip of Novint Falcon could follow the desired trajectory quickly with high accuracy in disturbance conditions. The proposed control could provide good performance for the haptic device to apply any robotics system.

\section{ACKNOWLEDGEMENTS}

This research was supported by Research Foundation funded by Thu Dau Mot University.

\section{REFERENCES}

[1] T. H. Massie and J. K. Salisbury, "The phantom haptic interface: A device for probing virtual objects," Proceedings of the ASME winter annual meeting, symposium on haptic interfaces for virtual environment and teleoperator systems, vol. 55, no. 1, pp. 295-300, 1994

[2] A. J. Silva, O. A. D. Ramirez, V. P. Vega, and J. P. O. Oliver, "Phantom omni haptic device: Kinematic and manipulability," 2009 Electronics, Robotics and Automotive Mechanics Conference (CERMA), 2009, pp. 193-198, doi: 10.1109/CERMA.2009.55.

[3] D. J. Block, M. B. Michelotti, and R. S. Sreenivas, "Application of the Novint Falcon haptic device as an actuator in real-time control," Paladyn, vol. 4, no. 3, pp. 182-193, 2013, doi: 10.2478/pjbr-2013-0017.

[4] D. Rácz, M. Takács, P. Galambos, and J. Somló, "A low-cost experimental device for compliant physical human-robot interaction," International Conference on Robotics in Alpe-Adria Danube Region, 2017, pp. 217-226, doi: 10.1007/978-3-31961276-8_25.

[5] H. Torres-Rodriguez, V. Parra-Vega, and F. Ruiz-Sanchez, "Integration of force-position control and haptic interface facilities for a virtual excavator simulator," Proceedings 12th International Conference on Advanced Robotics, ICAR'05, 2005, pp. 761-768, doi: 10.1109/ICAR.2005.1507494

[6] M. Wrock and S. Nokleby, "Haptic teleoperation of a manipulator using virtual fixtures and hybrid position-velocity control," IFToMM World Congress in Mechanism and Machine Science, 2011.

[7] P. Chotiprayanakul and D. Liu, "Workspace mapping and force control for small haptic device based robot teleoperation," 2009 International Conference on Information and Automation, 2009, pp. 1613-1618, doi: 10.1109/ICINFA.2009.5205175.

[8] D. Salle, F. Gosselin, P. Bidaud, and P. Gravez, "Analysis of haptic feedback performances in telesurgery robotic systems," Proceedings 10th IEEE International Workshop on Robot and Human Interactive Communication. ROMAN 2001 (Cat. No. 01TH8591), 2001, pp. 618-623, doi: 10.1109/ROMAN.2001.981973.

[9] Z. Xu, C. Song, and W. Wu, "Haptic tracking control for minimally invasive robotic surgery," Journal of biomedical engineering, vol. 29, no. 3, pp. 407-410, 2012.

[10] D. I. Ahlmark, H. Fredriksson, and K. Hyyppä, “Obstacle avoidance using haptics and a laser rangefinder," 2013 IEEE Workshop on Advanced Robotics and its Social Impacts, 2013, pp. 76-81, doi: 10.1109/ARSO.2013.6705509.

[11] G. S. Giri, Y. Maddahi, and K. Zareinia, "An application-based review of haptics technology,” Robotics, vol. 10, no. 1, 2021, Art. no. 29, doi: 10.3390/robotics10010029.

[12] M. Zarei, N. Kashi, A. Kalhor, M. T. Masouleh, and R. Systems, "Experimental study on shared-control of a mobile robot via a haptic device with an optimal velocity obstacle based receding horizon control approach," Journal of Intelligent \& Robotic Systems, vol. 97, no. 2, pp. 357-372, 2020, doi: 10.1007/s10846-019-01023-Z.

[13] F. Barontini, M. G. Catalano, L. Pallottino, B. Leporini, and M. Bianchi, "Integrating wearable haptics and obstacle avoidance for the visually impaired in indoor navigation: a user-centered approach," IEEE Transactions on Haptics, vol. 14, no. 1, pp. 109-122, 2020, doi: $10.1109 /$ TOH.2020.2996748.

[14] Z. Hou, P. Lu, and Z. Tu, "Nonsingular terminal sliding mode control for a quadrotor UAV with a total rotor failure," Aerospace Science Technology, vol. 98, 2020, Art. no. 105716, doi: 10.1016/j.ast.2020.105716.

[15] H. A. Malik, S. Rasool, A. Maqsood, and R. Riaz, "Effect of haptic feedback on pilot/operator performance during flight simulation," Applied Sciences, vol. 10, no. 11, 2020, Art. no. 3877, doi: 10.3390/app10113877.

[16] H. Courtois and N. Aouf, "Haptic feedback for obstacle avoidance applied to unmanned aerial vehicles," 2017 International Conference on Unmanned Aircraft Systems (ICUAS), 2017, pp. 417-424, doi: 10.1109/ICUAS.2017.7991328. 
[17] S. Fu, H. Saeidi, J. Huang, Y. Wang, and J. Wagner, "Customizable unmanned aerial vehicle haptic feedback interface: theory and test," Journal of Guidance, Control, Dynamics, vol. 41, no. 12, pp. 2581-2587, 2018, doi: 10.2514/1.G003645.

[18] A. Altan and R. Hacioğlu, "Model predictive control of three-axis gimbal system mounted on UAV for real-time target tracking under external disturbances," Mechanical Systems and Signal Processing, vol. 138, 2020, Art. no. 106548, doi: 10.1016/j.ymssp.2019.106548.

[19] S. Fu et al., "A haptic interface with adjustable feedback for unmanned aerial vehicles (UAVs)-model, control, and test," 2016 American Control Conference (ACC), 2016, pp. 467-472, doi: 10.1109/ACC.2016.7524958.

[20] N. Karbasizadeh, A. Aflakiyan, M. Zarei, M. T. Masouleh, and A. Kalhor, "Dynamic identification of the Novint Falcon haptic device," 2016 4th International Conference on Robotics and Mechatronics (ICROM), 2016, pp. 518-523, doi: 10.1109/ICRoM.2016.7886795.

[21] L.-W. Tsai and R. E. Stamper, "A parallel manipulator with only translational degrees of freedom," University of Maryland, College Park, vol. 301, pp. 1314-1328, 1997.

[22] F. Khadivar, S. Sadeghnejad, H. Moradi, G. Vossoughi, and F. Farahmand, "Dynamic characterization of a parallel haptic device for application as an actuator in a surgery simulator," 2017 5th RSI international conference on robotics and mechatronics (ICRoM), 2017, pp. 186-191, doi: 10.1109/ICRoM.2017.8466168.

[23] S. Martin and N. Hillier, "Characterisation of the Novint Falcon haptic device for application as a robot manipulator," Australasian Conference on Robotics and Automation (ACRA), 2009, pp. 291-292.

[24] M. R. Ghazali, M. Ahmad, and R. Ismail, "Data-driven neuroendocrine-PID controller design for twin rotor MIMO system," Journal of Physics: Conference Series, vol. 1529, 2020, Art. no. 042080, doi: 10.1088/1742-6596/1529/4/042080.

[25] A. Houari, I. Bachir, D. Mohamed, and M. Kara-Mohamed, "PID vs LQR controller for tilt rotor airplane," International Journal of Electrical Computer Engineering (IJECE), vol. 10, no. 6, pp. 6309-6318, 2020, doi: 10.11591/ijece.v10i6.pp6309-6318.

[26] D. Vega-Hernández, L. G. García-Valdovinos, O. A. Domínguez-Ramírez, and A. Curiel-Anaya, "Kinesthetic guidance based on PID control for haptic interaction," 2010 IEEE Electronics, Robotics and Automotive Mechanics Conference, 2010, pp. 624-630, doi: 10.1109/CERMA.2010.77.

[27] M. A. Choukou, S. Mbabaali, J. Bani Hani, and C. Cooke, "Haptic-enabled hand rehabilitation in stroke patients: A scoping review," Applied Sciences, vol. 11, no 8, 2021, Art. no. 38773712, doi: 10.3390/app11083712.

[28] N. Karbasizadeh, M. Zarei, A. Aflakian, M. T. Masouleh, and A. Kalhor, "Experimental dynamic identification and model feedforward control of Novint Falcon haptic device," Mechatronics, vol. 51, pp. 19-30, 2018, doi: 10.1016/j.mechatronics.2018.02.013. 